\title{
Adaptive Output Feedback Control for a Class of Stochastic Nonlinear Systems with SiISS Inverse Dynamics
}

\author{
Na Duan and Hai-Kuan Liu \\ School of Electrical Engineering and Automation, Jiangsu Normal University, Xuzhou 221116, China \\ Correspondence should be addressed to Na Duan, duanna08@163.com \\ Received 29 October 2011; Accepted 24 November 2011 \\ Academic Editor: Xue-Jun Xie \\ Copyright (c) 2012 N. Duan and H.-K. Liu. This is an open access article distributed under the \\ Creative Commons Attribution License, which permits unrestricted use, distribution, and \\ reproduction in any medium, provided the original work is properly cited. \\ The adaptive stabilization scheme based on tuning function for stochastic nonlinear systems with \\ stochastic integral input-to-state stability (SiISS) inverse dynamics is investigated. By combining \\ the stochastic LaSalle theorem and small-gain type conditions on SiISS, an adaptive output feed- \\ back controller is constructively designed. It is shown that all the closed-loop signals are bounded \\ almost surely and the stochastic closed-loop system is globally stable in probability.
}

\section{Introduction}

Global stabilization control design of stochastic nonlinear systems is one of the most important topics in nonlinear control theory, which has received and is increasingly receiving a great deal of attention; see, for example, [1-37] and the references therein. For a class of stochastic nonlinear systems with stochastic inverse dynamics, much progress has been made in the design of the global stabilization controller [12, 13, 15, 16, 24, 25, 31]. However, all these controllers are only robust against stochastic inverse dynamics with stringent stability margin. To weaken the stringent condition on stochastic inverse dynamics, a natural idea is to benefit from input-to-state stability (ISS) in [38] and integral input-to-state stability (iISS) in [39] which are now recognized as the central unifying concepts in feedback design and stability analysis of deterministic nonlinear systems. Tsinias in [21], Tang and Basar in [19] first proposed the concept of stochastic input-to-state stability (SISS) independently. Further in-depth study on SISS and its applications are presented in [9-11, 18, 22]. Motivated by these aforementioned important results, [34] showed that SISS condition can be weakened to stochastic integral input-to-state stability (SiISS) and developed a unifying output feedback framework for global regulation. 
Nonlinear small-gain theorem plays an important role in the controller design and stability analysis of deterministic nonlinear systems in [40,41]. While for stochastic nonlinear systems, there are fewer results on the small-gain theorem. [25] firstly established a gain-function-based stochastic nonlinear small-gain theorem for ISpS in probability. In some succeeding research work, $[9,11,35]$ presented some Lyapunov-based small-gain type conditions on SISS and SiISS, respectively. [4] further discusses the relationship of smallgain type conditions on SiISS and studies the problem of GAS in probability via output feedback.

In this paper, inspired by [4], a more general class of stochastic nonlinear systems with uncertain parameters and stochastic integral input-to-state stability (SiISS) inverse dynamics is investigated. By combining the stochastic LaSalle theorem and small-gain type conditions on SiISS, an adaptive output feedback controller is proposed to guarantee that all the closedloop signals are bounded almost surely and the stochastic closed-loop system is globally stable in probability.

The paper is organized as follows. Section 2 begins with the mathematical preliminaries. Section 3 presents statement of the problem. The design of adaptive output feedback controller is given in Section 4. The corresponding analysis is given in Section 5. Section 6 concludes the paper.

\section{Mathematical Preliminaries}

The following notations are used throughout the paper. $\mathbb{R}_{+}$stands for the set of all nonnegative real numbers, $\mathbb{R}^{n}$ is the $n$-dimensional Euclidean space, and $\mathbb{R}^{n \times m}$ is the space of real $n \times m$-matrices. For $x=\left(x_{1}, \ldots, x_{n}\right)$, one defines $\bar{x}_{i}=\left(x_{1}, \ldots, x_{i}\right), i=1, \ldots, n-1$. $C^{i}$ denotes the family of all the functions with continuous $i$ th partial derivatives. $L^{1}\left(\mathbb{R}_{+} ; \mathbb{R}_{+}\right)$is the family of all functions $l: \mathbb{R}_{+} \rightarrow \mathbb{R}_{+}$such that $\int_{0}^{\infty} l(t) d t<\infty$. For a given vector or matrix $X, X^{T}$ denotes its transpose, $\operatorname{Tr}\{X\}$ denotes its trace when $X$ is square. $|X|$ denotes the Euclidean norm of a vector $X$, and $\|X\|=\left(\operatorname{Tr}\left\{X X^{T}\right\}\right)^{1 / 2}$ for a matrix $X$. $\mathcal{K}$ denotes the set of all functions: $\mathbb{R}_{+} \rightarrow \mathbb{R}_{+}$, which are continuous, strictly increasing, and vanishing at zero; $\mathcal{K}_{\infty}$ is the set of all functions which are of class $\mathcal{K}$ and unbounded; $\mathcal{K} \perp$ denotes the set of all functions $\beta(s, t)$ : $\mathbb{R}_{+} \times \mathbb{R}_{+} \rightarrow \mathbb{R}_{+}$, which are of $\mathcal{K}$ for each fixed $t$ and decrease to zero as $t \rightarrow \infty$ for each fixed $s$.

Consider the stochastic nonlinear delay-free system

$$
d x=f(x, t) d t+g(x, t) d w, \quad \forall x(0)=x_{0} \in \mathbb{R}^{n},
$$

where $x \in \mathbb{R}^{n}, w$ is an $m$-dimensional standard Wiener process defined in a complete probability space $\left\{\Omega, \mathcal{F},\left\{\mathcal{F}_{t}\right\}_{t \geq 0}, P\right\}$ with $\Omega$ being a sample space, $\mathscr{F}$ being a $\sigma$-field, $\left\{\mathscr{F}_{t}\right\}_{t \geq 0}$ being a filtration, and $P$ being the probability measure. Borel measurable functions $f: \mathbb{R}^{n} \times$ $\mathbb{R}_{+} \rightarrow \mathbb{R}^{n}$ and $g: \mathbb{R}^{n} \times \mathbb{R}_{+} \rightarrow \mathbb{R}^{n \times m}$ are piecewise continuous in $t$ and locally bounded

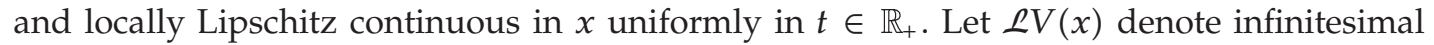
generator of function $V \in C^{2}$ along stochastic system (2.1) with the definition of

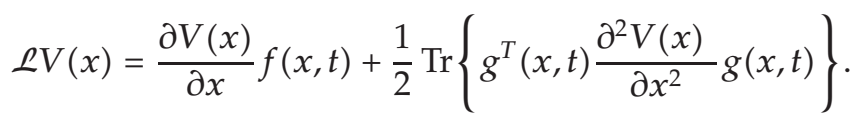


Definition 2.1 (see [34]). Stochastic process $\{\xi(t)\}_{t \geq t_{0}}$ is said to be bounded almost surely if $\sup _{t \geq t_{0}}|\xi(t)|<\infty$ a.s.

Lemma 2.2 (Stochastic LaSalle Theorem [14]). For system (2.1), if there exist functions $V \in C^{2}$, $\alpha_{1}, \alpha_{2} \in \mathcal{K}_{\infty}, l \in L^{1}\left(\mathbb{R}_{+} ; \mathbb{R}_{+}\right)$, and a continuous nonnegative function $W: \mathbb{R}^{n} \rightarrow \mathbb{R}$ such that for all $x \in \mathbb{R}^{n}, t \geq 0$,

$$
\alpha_{1}(|x|) \leq V(x) \leq \alpha_{2}(|x|), \quad \mathcal{L} V(x) \leq-W(x)+l(t),
$$

then for each $x_{0} \in \mathbb{R}^{n}$,

(i) system (2.1) has a unique strong solution on $[0, \infty)$, and solution $x(t)$ is bounded almost surely;

(ii) when $f(0, t) \equiv 0, g(0, t) \equiv 0, l(t) \equiv 0$, the equilibrium $x=0$ is globally stable in probability.

In the following, we cite two small-gain type conditions on SiISS in [35].

Consider the following stochastic nonlinear system

$$
d x=f(x, v, t) d t+g(x, v, t) d w, \quad \forall x(0)=x_{0} \in \mathbb{R}^{n},
$$

where $x \in \mathbb{R}^{n}$ is the state, $v \in \mathbb{R}^{m}$ is the input, and $w$ is an $r$-dimensional standard Wiener process defined as in (2.1). Borel measurable functions $f: \mathbb{R}^{n} \times \mathbb{R}^{m} \times \mathbb{R}_{+} \rightarrow \mathbb{R}^{n}$ and $g: \mathbb{R}^{n} \times$ $\mathbb{R}^{m} \times \mathbb{R}_{+} \rightarrow \mathbb{R}^{n \times r}$ are locally bounded and locally Lipschitz continuous with respect to $(x, v)$ uniformly in $t \in \mathbb{R}_{+}$.

Definition 2.3 (see [34]). System (2.4) is said to be stochastic integral input-to-state stable (SiISS) using Lyapunov function if there exist functions $V \in C^{2}\left(\mathbb{R}^{n} ; \mathbb{R}\right), \alpha_{1}, \alpha_{2}, \gamma \in \mathcal{K}_{\infty}$, and a merely positive definite continuous function $\alpha$ such that

$$
\alpha_{1}(|x|) \leq V(x) \leq \alpha_{2}(|x|), \quad\llcorner V(x) \leq-\alpha(|x|)+\gamma(|v|)
$$

The function $V$ satisfying (2.5) is said to be a SiISS-Lyapunov function, and $(\alpha, \gamma)$ in (2.5) is called the SiISS supply rate of system (2.4).

Lemma 2.4 (see [35]). For system (2.4) satisfying (2.5), if there exists a positive definite continuous function $\tilde{\alpha}$ such that $\limsup _{s \rightarrow 0_{+}} \tilde{\alpha}(s) / \alpha(s)<\infty, \limsup _{s \rightarrow \infty} \tilde{\alpha}(s) / \alpha(s)<\infty$, then there exists a function $\tilde{\gamma} \in \mathcal{K}_{\infty}$ such that $(\tilde{\alpha}, \tilde{\gamma})$ is a new SiISS supply rate of system (2.4). Moreover, if $\limsup _{s \rightarrow 0+} \gamma(s) / s^{m}<\infty$, then $\lim \sup _{s \rightarrow 0+} \tilde{\gamma}(s) / s^{m}<\infty$, where $m$ is any positive integer.

The following lemma shows that the condition at infinity can be removed if more prior information on stochastic system is known.

Assumption $H$. For functions $g, V, \alpha$ given in (2.4), (2.5) with $\liminf _{s \rightarrow \infty} \alpha(s)=\infty$, there exist known smooth positive definite functions $\phi_{1}, \phi_{2}$ such that $\|g(x, v, t)\| \leq \phi_{1}(|x|),|\partial V(x) / \partial x| \leq$ $\phi_{2}(|x|)$ and $\lim \sup _{s \rightarrow 0+} \phi_{1}^{2}(s) \phi_{2}^{2}(s) / \alpha(s)<\infty$. 
Lemma 2.5 (see [35]). For system (2.4) satisfying (2.5) and Assumption H, if there exists a positive definite function $\tilde{\alpha}$ such that

$$
\limsup _{s \rightarrow 0+} \frac{\tilde{\alpha}(s)}{\alpha(s)}<\infty, \quad \int_{0}^{\infty} e^{-\int_{0}^{s}\left(1 / \zeta\left(\alpha_{1}^{-1}(\tau)\right)\right) d \tau}\left[\xi\left(\alpha_{1}^{-1}(s)\right)\right]^{\prime} d s<\infty
$$

where $\xi(\cdot) \geq 0, \zeta(\cdot)>0$ are smooth increasing functions with $\xi(s) \alpha(s) \geq 2 \tilde{\alpha}(s), \zeta(s) \alpha(s) \geq$ $\phi_{1}^{2}(s) \phi_{2}^{2}(s)$ for any $s \geq 0$, then there exists a function $\tilde{\gamma} \in \mathcal{K}_{\infty}$ such that $(\tilde{\alpha}, \tilde{\gamma})$ is a new SiISS supply rate of system (2.4). Moreover, if $\lim \sup _{s \rightarrow 0_{+}} \gamma(s) / s^{m}<\infty$, then $\lim \sup _{s \rightarrow 0+} \tilde{\gamma}(s) / s^{m}<\infty$, where $m$ is any positive integer.

Lemma 2.6. Let $x, y$ be real variables, then for any positive integers $m, n$ and continuous function $a(\cdot) \geq 0, a(\cdot) x^{m} y^{n} \leq b|x|^{m+n}+(n /(m+n))((m+n) / m)^{-m / n} a^{(m+n) / n}(\cdot) b^{m / n}|y|^{m+n}$, where $b>0$ is any real number.

\section{Problem Statement}

In this paper, we consider a class of stochastic nonlinear systems described by

$$
\begin{gathered}
d \eta=\varphi_{0}\left(\eta, x_{1}\right) d t+\psi_{0}\left(\eta, x_{1}\right) d w, \\
d x_{1}=x_{2} d t+\varphi_{1}(\eta, x) d t+\psi_{1}(\eta, x) d w, \\
\vdots \\
d x_{n-1}=x_{n} d t+\varphi_{n-1}(\eta, x) d t+\psi_{n-1}(\eta, x) d w, \\
d x_{n}=u d t+\varphi_{n}(\eta, x) d t+\psi_{n}(\eta, x) d w, \\
y=x_{1},
\end{gathered}
$$

where $\left(x_{2}, \ldots, x_{n}\right) \in \mathbb{R}^{n-1}, u, y \in \mathbb{R}$ represent the unmeasurable state, the control input, and the measurable output, respectively. $\eta \in \mathbb{R}^{n_{0}}$ is referred to as the stochastic inverse dynamics. The initial value $\left(\eta^{T}(0), x_{1}(0), \ldots, x_{n}(0)\right)$ can be chosen arbitrarily. $w$ is an $m$-dimensional standard Wiener process defined as in (2.1). Uncertain functions $\varphi_{i}: \mathbb{R}^{n_{0}} \times \mathbb{R}^{n} \rightarrow \mathbb{R}, \psi_{i}$ : $\mathbb{R}^{n_{0}} \times \mathbb{R}^{n} \rightarrow \mathbb{R}^{m}, 1 \leq i \leq n$, are smooth functions. It is assumed that $\varphi_{0}$ and $\psi_{0}$ are locally Lipschitz continuous functions.

The research purpose of this paper is to design an adaptive output feedback controller for system (3.1) by using stochastic LaSalle theorem and small-gain type conditions on SiISS, in such a way that, for all initial conditions, the solutions of the closed-loop system are bounded almost surely and the closed-loop systems are globally stable in probability. To achieve the control purpose, we need the following assumptions.

Assumption 3.1. For each $1 \leq i \leq n$, there exist the unknown constant $\theta_{i}>0$, the known nonnegative smooth functions $\varphi_{i 0}, \varphi_{i 1}, \psi_{i 0}$ and $\psi_{i 1}$ with $\varphi_{i j}(0)=0, \psi_{i j}(0)=0, j=0,1$, such that $\left|\varphi_{i}(\eta, x)\right| \leq \theta_{i}\left(\varphi_{i 0}(|\eta|)+\varphi_{i 1}\left(x_{1}\right)\right),\left|\psi_{i}(\eta, x)\right| \leq \theta_{i}\left(\psi_{i 0}(|\eta|)+\psi_{i 1}\left(x_{1}\right)\right)$. 
For Assumption 3.1, there exist smooth functions $\bar{\varphi}_{i 1}$ and $\bar{\psi}_{i 1}$ satisfying

$$
\varphi_{i 1}\left(x_{1}\right)=x_{1} \bar{\varphi}_{i 1}\left(x_{1}\right), \quad \psi_{i 1}\left(x_{1}\right)=x_{1} \bar{\psi}_{i 1}\left(x_{1}\right),
$$

which will be frequently used in the subsequent sections.

Assumption 3.2. For the $\eta$-subsystem, there exists an SiISS-Lyapunov function $V_{0}(\eta)$. Namely, $V_{0}$ satisfies $\underline{\alpha}(|\eta|) \leq V_{0}(\eta) \leq \bar{\alpha}(|\eta|), \mathcal{L} V_{0}(\eta) \leq-\alpha(|\eta|)+\gamma\left(\left|x_{1}\right|\right)$, where $\underline{\alpha}, \bar{\alpha}, \gamma$ are class $\mathcal{K}_{\infty}$ functions, and $\alpha$ is merely a continuous positive definite function.

\section{Design of an Adaptive Output Feedback Controller}

\subsection{Reduced-Order Observer Design}

Introduce the following reduced-order observer:

$$
\begin{gathered}
\dot{\hat{x}}_{i}=\widehat{x}_{i+1}+k_{i+1} y-k_{i}\left(\widehat{x}_{1}+k_{1} y\right), \quad 1 \leq i \leq n-2, \\
\dot{\hat{x}}_{n-1}=u-k_{n-1}\left(\widehat{x}_{1}+k_{1} y\right),
\end{gathered}
$$

where $k=\left(k_{1}, \ldots, k_{n-1}\right)^{T}$ is chosen such that $A_{0}=\left[\begin{array}{cc}I_{n-2} \\ -k & 0 . .0\end{array}\right]$ is asymptotically stable. Define the error variable

$$
e_{i}=\frac{1}{\theta^{*}}\left(x_{i+1}-\widehat{x}_{i}-k_{i} x_{1}\right), \quad 1 \leq i \leq n-1, \theta^{*}=\max \left\{1, \theta_{1}, \ldots, \theta_{n}\right\}
$$

By (3.1), (4.1), and (4.2), one gets

$$
d e_{i}=\left(e_{i+1}-k_{i} e_{1}\right) d t+\frac{1}{\theta^{*}}\left(\varphi_{i+1}(\eta, x)-k_{i} \varphi_{1}(\eta, x)\right) d t+\frac{1}{\theta^{*}}\left(\psi_{i+1}(\eta, x)-k_{i} \psi_{1}(\eta, x)\right) d w,
$$

whose compact form is

$$
d e=\left(A_{0} e+\Phi(\eta, x)\right) d t+\Psi(\eta, x) d w,
$$

where

$$
\begin{gathered}
e=\left(e_{1}, \ldots, e_{n-1}\right)^{T}, \\
\Phi(\eta, x)=\frac{1}{\theta^{*}}\left(\varphi_{2}(\eta, x)-k_{1} \varphi_{1}(\eta, x), \varphi_{3}(\eta, x)-k_{2} \varphi_{1}(\eta, x), \ldots, \varphi_{n}(\eta, x)-k_{n-1} \varphi_{1}(\eta, x)\right)^{T}, \\
\Psi(\eta, x)=\frac{1}{\theta^{*}}\left(\psi_{2}(\eta, x)-k_{1} \psi_{1}(\eta, x), \psi_{3}(\eta, x)-k_{2} \psi_{1}(\eta, x), \ldots, \psi_{n}(\eta, x)-k_{n-1} \psi_{1}(\eta, x)\right)^{T} .
\end{gathered}
$$




\subsection{The Design of Adaptive Backstepping Controller}

From (3.1), (4.1), (4.2), and (4.4), the interconnected system is represented as

$$
\begin{gathered}
d \eta=\varphi_{0}(\eta, y) d t+\psi_{0}(\eta, y) d w, \\
d e=\left(A_{0} e+\Phi(\eta, x)\right) d t+\Psi(\eta, x) d w, \\
d y=\left(\widehat{x}_{1}+k_{1} y+\theta^{*} e_{1}+\varphi_{1}(\eta, x)\right) d t+\psi_{1}(\eta, x) d w, \\
d \widehat{x}_{1}=\left(\widehat{x}_{2}+k_{2} y-k_{1}\left(\widehat{x}_{1}+k_{1} y\right)\right) d t \\
d \widehat{x}_{2}=\left(\widehat{x}_{3}+k_{3} y-k_{2}\left(\widehat{x}_{1}+k_{1} y\right)\right) d t \\
\vdots \\
d \widehat{x}_{n-1}=\left(u-k_{n-1}\left(\widehat{x}_{1}+k_{1} y\right)\right) d t .
\end{gathered}
$$

Next, we will develop an adaptive backstepping controller by using the backstepping method. Firstly, a coordinate transformation is introduced

$$
z_{1}=y, \quad z_{i+1}=\widehat{x}_{i}-\alpha_{i}\left(y, \widehat{x}_{1}, \ldots, \widehat{x}_{i-1}, \widehat{\theta}\right), \quad i=1, \ldots, n-1
$$

Step 1. By (4.6) and (4.7), one has

$$
d z_{1}=\left(\alpha_{1}+z_{2}+k_{1} y+\theta^{*} e_{1}+\varphi_{1}(\eta, x)\right) d t+\psi_{1}(\eta, x) d w .
$$

Since $A_{0}$ is asymptotically stable, there exists a positive definite matrix $P$ such that $P A_{0}+$ $A_{0}^{T} P=-I_{n-1}$. Choose

$$
V_{1}\left(e, z_{1}, \widehat{\theta}\right)=\frac{\delta}{2}\left(e^{T} P e\right)^{2}+\frac{1}{2 \Gamma} \tilde{\theta}^{2}+\frac{1}{4} z_{1}^{4}, \quad \delta>0, \Gamma>0
$$

where $\tilde{\theta}=\widehat{\theta}-\theta, \widehat{\theta}$ is the estimate of $\theta=\max \left\{\theta^{*}, \theta^{*(4 / 3)}, \theta^{* 4}\right\}$. In view of (2.2), (4.4), (4.8), and (4.9), then

$$
\begin{aligned}
\mathcal{L} V_{1}= & 2 \delta e^{T} \operatorname{Pee}^{T} P\left(A_{0} e+\Phi(\eta, x)\right)+\frac{1}{2} \operatorname{Tr}\left\{\Psi^{T}(\eta, x)\left(4 \delta\left(e^{T} P\right)^{T} e^{T} P+2 \delta e^{T} P e P\right) \Psi(\eta, x)\right\} \\
& +\frac{1}{\Gamma} \tilde{\theta} \dot{\theta}+z_{1}^{3}\left(\alpha_{1}+z_{2}+k_{1} y+\theta^{*} e_{1}+\varphi_{1}(\eta, x)\right)+\frac{3}{2} z_{1}^{2} \operatorname{Tr}\left\{\psi_{1}^{T}(\eta, x) \psi_{1}(\eta, x)\right\}
\end{aligned}
$$


Mathematical Problems in Engineering

$$
\begin{aligned}
= & \delta e^{T} P e e^{T}\left(P A_{0}+A_{0}^{T} P\right) e+2 \delta e^{T} P e e^{T} P \Phi(\eta, x) \\
& +\operatorname{Tr}\left\{\Psi^{T}(\eta, x)\left(2 \delta\left(e^{T} P\right)^{T} e^{T} P+\delta e^{T} P e P\right) \Psi(\eta, x)\right\} \\
& +\frac{1}{\Gamma} \tilde{\theta} \dot{\theta}+z_{1}^{3}\left(\alpha_{1}+z_{2}+k_{1} y+\theta^{*} e_{1}+\varphi_{1}(\eta, x)\right)+\frac{3}{2} z_{1}^{2} \operatorname{Tr}\left\{\psi_{1}^{T}(\eta, x) \psi_{1}(\eta, x)\right\} \\
\leq & -\delta \lambda_{\min }(P)|e|^{4}+2 \delta\|P\|^{2}|e|^{3}|\Phi(\eta, x)|+3 \delta\|P\|^{2}|e|^{2}\|\Psi(\eta, x)\|^{2}+\frac{1}{\Gamma} \tilde{\theta} \dot{\hat{\theta}} \\
& +z_{1}^{3}\left(\alpha_{1}+z_{2}+k_{1} y+\theta^{*} e_{1}+\varphi_{1}(\eta, x)\right)+\frac{3}{2} z_{1}^{2} \operatorname{Tr}\left\{\psi_{1}^{T}(\eta, x) \psi_{1}(\eta, x)\right\} .
\end{aligned}
$$

Applying Assumption 3.1, (3.2), (4.3), (4.7), and Lemma 2.6, it follows that

$$
\begin{gathered}
2 \delta\|P\|^{2}|e|^{3} \Phi|(\eta, x)| \leq a_{01}|e|^{4}+\bar{a}_{01}|\Phi(\eta, x)|^{4}, \\
3 \delta\|P\|^{2}|e|^{2}\|\Psi(\eta, x)\|^{2} \leq a_{02}|e|^{4}+\bar{a}_{02}\|\Psi(\eta, x)\|^{4}, \\
z_{1}^{3} z_{2} \leq a_{10} z_{1}^{4}+\bar{a}_{10} z_{2}^{4}, \quad \theta^{*} z_{1}^{3} e_{1} \leq a_{11} e_{1}^{4}+\theta^{*(4 / 3)} \gamma_{11}\left(z_{1}\right) z_{1}^{4} \leq a_{11} e_{1}^{4}+\theta \gamma_{11}\left(z_{1}\right) z_{1}^{4}, \\
z_{1}^{3} \varphi_{1}(\eta, x) \leq\left|z_{1}\right|^{3} \theta^{*}\left(\varphi_{10}(|\eta|)+\varphi_{11}\left(z_{1}\right)\right) \leq a_{12} \varphi_{10}^{4}(|\eta|)+\theta \gamma_{12}\left(z_{1}\right) z_{1}^{4}, \\
\frac{3}{2} z_{1}^{2} \operatorname{Tr}\left\{\psi_{1}^{T}(\eta, x) \psi_{1}(\eta, x)\right\} \leq 3 z_{1}^{2} \theta^{* 2}\left(\psi_{10}^{2}(|\eta|)+\psi_{11}^{2}\left(z_{1}\right)\right) \leq a_{13} \psi_{10}^{4}(|\eta|)+\theta \gamma_{13}\left(z_{1}\right) z_{1}^{4},
\end{gathered}
$$

where $\bar{a}_{01}, \bar{a}_{02}, \gamma_{11}, \gamma_{12}$, and $\gamma_{13}$ are smooth functions, $a_{01}, a_{02}, a_{10}, \bar{a}_{10}, a_{11}, a_{12}, a_{13}>0$ are constants.

Using $\left(a_{1}+\cdots+a_{n}\right)^{2} \leq(x+a)^{n}=n \sum_{i=1}^{n} a_{i}^{2},(a+b)^{4} \leq 8 a^{4}+8 b^{4}, y=x_{1}$, Assumption 3.1, (3.2), and (4.5), one gets

$$
\begin{gathered}
|\Phi(\eta, x)|^{4} \leq 64(n-1)\left(\left(k_{1}^{4}+\cdots+k_{n-1}^{4}\right)\left(\varphi_{10}^{4}+y^{4} \bar{\varphi}_{11}^{4}\right)+\varphi_{20}^{4}+\cdots+\varphi_{n 0}^{4}+y^{4}\left(\bar{\varphi}_{21}^{4}+\cdots+\bar{\varphi}_{n 1}^{4}\right)\right), \\
\|\Psi(\eta, x)\|^{4} \leq 64(n-1)\left(\left(k_{1}^{4}+\cdots+k_{n-1}^{4}\right)\left(\psi_{10}^{4}+y^{4} \bar{\psi}_{11}^{4}\right)+\psi_{20}^{4}+\cdots+\psi_{n 0}^{4}+y^{4}\left(\bar{\psi}_{21}^{4}+\cdots+\bar{\psi}_{n 1}^{4}\right)\right) .
\end{gathered}
$$

Substituting (4.11)-(4.12) into (4.10) and using $z_{1}=y$ lead to

$$
\begin{aligned}
\mathcal{L} V_{1} \leq & -a_{00}|e|^{4}+a_{11} e_{1}^{4}+\Delta_{1}(|\eta|)+\bar{a}_{10} z_{2}^{4}+z_{1}^{3}\left(\alpha_{1}+k_{1} z_{1}+\Delta_{00}\left(z_{1}\right) z_{1}+a_{10} z_{1}\right) \\
& +\theta\left(\gamma_{11}\left(z_{1}\right)+\gamma_{12}\left(z_{1}\right)+\gamma_{13}\left(z_{1}\right)\right) z_{1}^{4}+\frac{1}{\Gamma} \tilde{\theta} \dot{\hat{\theta}}
\end{aligned}
$$


where

$$
\begin{aligned}
a_{00}= & \delta \lambda_{\min }(P)-a_{01}-a_{02} \\
\Delta_{1}(|\eta|)= & 64(n-1) \bar{a}_{01}\left(\left(k_{1}^{4}+\cdots+k_{n-1}^{4}\right) \varphi_{10}^{4}(|\eta|)+\varphi_{20}^{4}(|\eta|)+\cdots+\varphi_{n 0}^{4}(|\eta|)\right) \\
& +64(n-1) \bar{a}_{02}\left(\left(k_{1}^{4}+\cdots+k_{n-1}^{4}\right) \psi_{10}^{4}(|\eta|)+\psi_{20}^{4}(|\eta|)+\cdots+\psi_{n 0}^{4}(|\eta|)\right) \\
& +a_{12} \varphi_{10}^{4}(|\eta|)+a_{13} \psi_{10}^{4}(|\eta|) \\
\Delta_{00}\left(z_{1}\right)= & 64(n-1) \bar{a}_{01}\left(\left(k_{1}^{4}+\cdots+k_{n-1}^{4}\right) \bar{\varphi}_{11}^{4}\left(z_{1}\right)+\bar{\varphi}_{21}^{4}\left(z_{1}\right)+\cdots+\bar{\varphi}_{n 1}^{4}\left(z_{1}\right)\right) \\
& +64(n-1) \bar{a}_{02}\left(\left(k_{1}^{4}+\cdots+k_{n-1}^{4}\right) \bar{\psi}_{11}^{4}\left(z_{1}\right)+\bar{\psi}_{21}^{4}\left(z_{1}\right)+\cdots+\bar{\psi}_{n 1}^{4}\left(z_{1}\right)\right) .
\end{aligned}
$$

Choosing the virtual control $\alpha_{1}(\cdot)$ and the tuning function $\tau_{1}(\cdot)$

$$
\begin{gathered}
\alpha_{1}(y, \widehat{\theta})=-z_{1}\left(c_{1}+v_{1}\left(z_{1}\right)+k_{1}+\Delta_{00}\left(z_{1}\right)+a_{10}+\widehat{\theta}\left(\gamma_{11}\left(z_{1}\right)+\gamma_{12}\left(z_{1}\right)+\gamma_{13}\left(z_{1}\right)\right)\right) \\
\tau_{1}(y)=\Gamma\left(\gamma_{11}\left(z_{1}\right)+\gamma_{12}\left(z_{1}\right)+\gamma_{13}\left(z_{1}\right)\right) z_{1}^{4}
\end{gathered}
$$

one gets

$$
\mathcal{L} V_{1} \leq-c_{1} z_{1}^{4}-v_{1}\left(z_{1}\right) z_{1}^{4}-a_{00}|e|^{4}+a_{11} e_{1}^{4}+\Delta_{1}(|\eta|)+\bar{a}_{10} z_{2}^{4}+\frac{1}{\Gamma} \tilde{\theta}\left(\dot{\hat{\theta}}-\tau_{1}\right)
$$

where $c_{1}>0$ is design parameter and $v_{1}\left(z_{1}\right)>0$ is a smooth function to be chosen later.

Step $i(i=2, \ldots, n)$. For notation coherence, denote $u=\alpha_{n}, z_{n+1}=0$. At this step, we can obtain a property similar to (4.16), which is presented by the following lemma.

Lemma 4.1. For the ith Lyapunov function $V_{i}\left(e, \bar{z}_{i}, \widehat{\theta}\right)=(\delta / 2)\left(e^{T} P e\right)^{2}+(1 / 2 \Gamma) \tilde{\theta}^{2}+\sum_{j=1}^{i}\left(z_{j}^{4} / 4\right)$, there are the virtual control law $\alpha_{i}\left(y, \widehat{x}_{1}, \ldots, \widehat{x}_{i-1}, \widehat{\theta}\right)$ and the tuning function $\tau_{i}$ with the form

$$
\begin{gathered}
\begin{array}{c}
\alpha_{i}\left(y, \widehat{x}_{1}, \ldots, \widehat{x}_{i-1}, \hat{\theta}\right)=-\Omega_{i}-z_{i}\left(c_{i}+\bar{a}_{i-1,0}+a_{i 0}+\widehat{\theta}\left(\gamma_{i 1}+\gamma_{i 2}+\gamma_{i 3}+\gamma_{i 4}\right)\right. \\
\left.+\Gamma\left(\gamma_{i 1}+\gamma_{i 2}+\gamma_{i 3}+\gamma_{i 4}\right) \sum_{j=1}^{i-1} \frac{\partial \alpha_{j}}{\partial \widehat{\theta}} z_{j+1}^{3}\right)+\frac{\partial \alpha_{i-1}}{\partial \widehat{\theta}} \tau_{i},
\end{array} \\
\tau_{i}\left(y, \widehat{x}_{1}, \ldots, \widehat{x}_{i-1}, \widehat{\theta}\right)=\tau_{i-1}+\Gamma\left(\gamma_{i 1}+\gamma_{i 2}+\gamma_{i 3}+\gamma_{i 4}\right) z_{i}^{4},
\end{gathered}
$$


such that

$$
\begin{aligned}
\mathcal{L} V_{i} \leq & -\sum_{j=1}^{i} c_{j} z_{j}^{4}+\sum_{j=2}^{i} b_{j} z_{1}^{4}-v_{1}\left(z_{1}\right) z_{1}^{4}-a_{00}|e|^{4}+\sum_{j=1}^{i} a_{j 1} e_{1}^{4}+\Delta_{i}(|\eta|)+\bar{a}_{i 0} z_{i+1}^{4} \\
& +\left(\frac{1}{\Gamma} \widetilde{\theta}-\sum_{j=1}^{i-1} \frac{\partial \alpha_{j}}{\partial \widehat{\theta}} z_{j+1}^{3}\right)\left(\dot{\hat{\theta}}-\tau_{i}\right)
\end{aligned}
$$

where $c_{i}>0$ is the designed parameters, $a_{i 0}, \bar{a}_{i-1,0}, a_{i 1}, b_{i}$ are some positive constants, $\gamma_{i 1}, \gamma_{i 2}, \gamma_{i 3}, \gamma_{i 4}$ are smooth nonnegative functions, whose choices are given in the proof.

Proof. See the appendix.

Therefore, at the end of the recursive procedure, the controller can be constructed as

$$
u=\alpha_{n}\left(y, \widehat{x}_{1}, \ldots, \widehat{x}_{n-1}, \widehat{\theta}\right), \quad \dot{\hat{\theta}}=\tau_{n}\left(y, \widehat{x}_{1}, \ldots, \widehat{x}_{n-1}, \widehat{\theta}\right) .
$$

Choosing parameters $\delta, a_{01}, a_{02}, a_{11}, \ldots, a_{n 1}, c_{2}, \ldots, c_{n}$ to satisfy

$$
a_{0}=\delta \lambda_{\min }(P)-a_{01}-a_{02}-\sum_{i=1}^{n} a_{i 1}>0, \quad c_{2}, \ldots, c_{n}>0,
$$

by (4.16) and (4.18), one has

$$
\mathcal{L} V_{n} \leq-\sum_{i=1}^{n} c_{i} z_{i}^{4}+\sum_{j=2}^{n} b_{j} z_{1}^{4}-v_{1}\left(z_{1}\right) z_{1}^{4}-a_{0}|e|^{4}+\Delta_{n}(|\eta|),
$$

where

$$
\begin{aligned}
V_{n}(e, z, \widehat{\theta})= & \frac{\delta}{2}\left(e^{T} P e\right)^{2}+\frac{1}{2 \Gamma} \widetilde{\theta}^{2}+\frac{1}{4} \sum_{i=1}^{n} z_{i}^{4} \\
\Delta_{n}(|\eta|)= & 64(n-1) \bar{a}_{01}\left(\left(k_{1}^{4}+\ldots+k_{n-1}^{4}\right) \varphi_{10}^{4}(|\eta|)+\varphi_{20}^{4}(|\eta|)+\ldots+\varphi_{n 0}^{4}(|\eta|)\right) \\
& +64(n-1) \bar{a}_{02}\left(\left(k_{1}^{4}+\ldots+k_{n-1}^{4}\right) \psi_{10}^{4}(|\eta|)+\psi_{20}^{4}(|\eta|)+\ldots+\psi_{n 0}^{4}(|\eta|)\right) \\
& +\sum_{i=1}^{n}\left(a_{i 2} \varphi_{10}^{4}(|\eta|)+a_{i 3} \psi_{10}^{4}(|\eta|)\right)+\sum_{j=2}^{n} a_{j 4} \psi_{10}^{4}(|\eta|) .
\end{aligned}
$$

\section{Stability Analysis}

We state the main theorems in this paper. This section is divided into two parts. 


\subsection{Case I}

Theorem 5.1. Assume that Assumptions 3.1 and 3.2 hold with the following properties:

$$
\limsup _{s \rightarrow 0+} \frac{\Delta_{n}(|\eta|)}{\alpha(s)}<\infty, \quad \limsup _{s \rightarrow \infty} \frac{\Delta_{n}(|\eta|)}{\alpha(s)}<\infty
$$

If lim sup ${ }_{s \rightarrow 0_{+}} \gamma(s) / s^{4}<\infty$ in Assumption 3.2, by appropriately choosing the positive smooth function $v_{1}(\cdot)$ in (4.15) and the parameters $\delta, a_{01}, a_{02}, a_{11}, \ldots, a_{n 1}, c_{2}, \ldots, c_{n}$ to satisfy (4.20), then

(i) the closed-loop system consisting of (3.1), (4.1), (4.2), (4.7), (4.15), (4.17), and (4.19) has a unique and almost surely bounded strong solution on $[0, \infty)$;

(ii) for each initial value $(\eta(0), x(0), \widehat{x}(0), \widehat{\theta}(0))$, the equilibrium $(\eta, x)=(0,0)$ is globally stable in probability, where $\widehat{x}=\left(\widehat{x}_{1}, \ldots, \widehat{x}_{n-1}\right)$.

Proof. For any constant $\epsilon>0$, by (5.1), one has

$$
\limsup _{s \rightarrow 0+} \frac{(1+\epsilon) \Delta_{n}(|\eta|)}{\alpha(s)}<\infty, \quad \limsup _{s \rightarrow \infty} \frac{(1+\epsilon) \Delta_{n}(|\eta|)}{\alpha(s)}<\infty
$$

For the $\eta$-subsystem, by Lemma 2.4, there exists a positive and radially unbounded Lyapunov function $\tilde{V}_{0}(\eta) \in C^{2}$ and $\tilde{\gamma}=\rho \gamma$ such that

$$
\begin{gathered}
\mathcal{L} \tilde{V}_{0}(\eta) \leq-(1+\epsilon) \Delta_{n}(|\eta|)+\tilde{\gamma}\left(\left|z_{1}\right|\right), \\
\limsup _{s \rightarrow 0+} \frac{\tilde{\gamma}(s)}{s^{4}}<\infty,
\end{gathered}
$$

where $\rho$ is a positive constant satisfying $(1+\epsilon) \Delta_{n}(|\eta|) \leq \rho \alpha(s)$ for all $s \geq 0$. Choosing the following Lyapunov function for the entire closed-loop system

$$
V(\eta, e, z, \widehat{\theta})=\tilde{V}_{0}(\eta)+V_{n}(e, z, \hat{\theta})
$$

and combining (4.21) and (5.3), one obtains

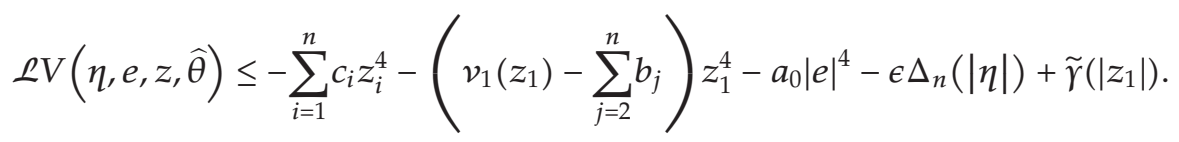

By (5.4), there always exists a smooth function $v_{1}(\cdot)$ to satisfy the following two inequalities:

$$
z_{1}^{4}\left(v_{1}\left(z_{1}\right)-\sum_{j=2}^{n} b_{j}\right) \geq \tilde{\gamma}\left(\left|z_{1}\right|\right), \quad v_{1}\left(z_{1}\right) \geq \sum_{j=2}^{n} b_{j}
$$


Substituting (5.7) into (5.6) leads to

$$
\begin{aligned}
\varrho V(\eta, e, z, \hat{\theta}) & \leq-\sum_{i=1}^{n} c_{i} z_{i}^{4}-a_{0}|e|^{4}-\epsilon \Delta_{n}(|\eta|) \\
& \triangleq-W(\eta, e, z) .
\end{aligned}
$$

Noting that $\varphi_{i 0}(\cdot)$ and $\psi_{i 0}(\cdot)$ are nonnegative smooth functions and using (4.22), it follows that $\Delta_{n}(\cdot)$ and $W(\cdot)$ are continuous nonnegative functions. By (5.5), (5.8), and Lemma 2.2, one concludes that all the solutions of the closed-loop system are bounded almost surely, the equilibrium $(\eta, e, z)=(0,0,0)$ is globally stable in probability. By $(3.1),(4.1),(4.2),(4.7)$, (4.15), (4.17), (4.19), and the almost sure boundedness of all the signals, it is not difficult to recursively prove that the equilibrium $(\eta, x)=(0,0)$ is globally stable in probability.

\subsection{Case II}

If more information about $\alpha$ in Assumption 3.2 is known, that is, $\lim _{\inf } f_{\infty} \alpha(s)=\infty$, further results under the weaker conditions is given as follows.

Assumption 5.2. For functions $\psi_{0}$ and $V_{0}$ given by (4.22) and Assumption 3.2, there exist known smooth nonnegative functions $\widetilde{\psi}_{1}$ and $\widetilde{\psi}_{2}$ with $\tilde{\psi}_{1}(0)=\tilde{\psi}_{2}(0)=0$, such that $\left\|\psi_{0}\left(\eta, x_{1}\right)\right\| \leq \widetilde{\psi}_{1}(|\eta|)$ and $\left|\partial V_{0}(\eta) / \partial \eta\right| \leq \widetilde{\psi}_{2}(|\eta|)$.

Lemma 5.3. For $\Delta_{n}, \underline{\alpha}, \alpha, \widetilde{\psi}_{1}$ and $\tilde{\psi}_{2}$ given by (4.22), Assumptions 3.2 and 5.2, if

$$
\limsup _{s \rightarrow 0+} \frac{\Delta_{n}(s)+\tilde{\psi}_{1}^{2}(s) \tilde{\psi}_{2}^{2}(s)}{\alpha(s)}<\infty, \quad \int_{0}^{\infty} e^{-\int_{0}^{s}\left(1 / \zeta_{1}\left(\underline{\alpha}^{-1}(\tau)\right)\right) d \tau}\left[\xi_{1}\left(\underline{\alpha}^{-1}(s)\right)\right]^{\prime} d s<\infty
$$

where $\xi_{1}(\cdot) \geq 0$ and $\zeta_{1}(\cdot)>0$ are smooth increasing functions satisfying

$$
\xi_{1}(s) \alpha(s) \geq 2(1+\epsilon) \Delta_{n}(s), \quad \zeta_{1}(s) \alpha(s) \geq \tilde{\psi}_{1}^{2}(s) \tilde{\psi}_{2}^{2}(s), \quad \forall s \geq 0,
$$

$\epsilon$ is any positive constant. Then there exists a function $\tilde{\gamma} \in \mathcal{K}_{\infty}$ such that $\left((1+\epsilon) \Delta_{n}, \tilde{\gamma}\right)$ is a new SiISS supply rate of the $\eta$-subsystem in (3.1). Moreover, if $\gamma$ in Assumptions 3.2 satisfies $\lim \sup _{s \rightarrow 0+} \gamma(s) / s^{4}<\infty$, then $\lim \sup _{s \rightarrow 0+} \tilde{\gamma}(s) / s^{4}<\infty$.

Since the condition (5.9) is weaker than (5.1), by using Lemma 5.3, we give further results under the weaker condition (5.9).

Theorem 5.4. Suppose that Assumptions 3.1-5.2 and the conditions of Lemma 5.3 hold. If $\lim \sup _{s \rightarrow 0+} \gamma(s) / s^{4}<\infty$ in Assumption 3.2, by appropriately choosing the positive smooth function $v_{1}(\cdot)$ in (4.15) and the parameters $\delta, a_{01}, a_{02}, a_{11}, \ldots, a_{n 1}, c_{2}, \ldots, c_{n}$ to satisfy (4.20), then

(1) the closed-loop system consisting of (3.1), (4.1), (4.2), (4.7), (4.15), (4.17), and (4.19) has a unique and almost surely bounded strong solution on $[0, \infty)$;

(2) for each initial value $(\eta(0), x(0), \widehat{x}(0), \widehat{\theta}(0))$, the equilibrium $(\eta, x)=(0,0)$ is globally stable in probability. 


\section{Conclusions}

This paper further considers a more general class of stochastic nonlinear systems with uncertain parameters and SiISS inverse dynamics. By combining the stochastic LaSalle theorem and small-gain type conditions on SiISS, an adaptive output feedback controller is designed to guarantee that all the closed-loop signals are bounded almost surely and the stochastic closed-loop system is globally stable in probability.

There are two remaining problems to be investigated: (1) an essential problem is to find a practical example with explicit physical meaning for system (3.1). A preliminary attempt on high-order stochastic nonlinear system can be found in [28]. (2) How to design an output feedback controller by using this method in this paper for system (3.1) in which the drift and diffusion vector fields depend on the unmeasurable states besides the measurable output?

\section{Appendix}

Proof of Lemma 4.1. We prove Lemma 4.1 by induction. Assume that at Step $i-1$, there are virtual control laws

$$
\begin{aligned}
\alpha_{i-1}\left(y, \widehat{x}_{1}, \ldots, \widehat{x}_{i-2}, \hat{\theta}\right)= & -\Omega_{i-1}-z_{i-1} \\
& \times\left(c_{i-1}+\bar{a}_{i-2,0}+a_{i-1,0}+\bar{a}_{i-1,1}+\widehat{\theta}\left(\gamma_{i-1,1}+\gamma_{i-1,2}+\gamma_{i-1,3}+\gamma_{i-1,4}\right)\right. \\
& \left.+\Gamma\left(\gamma_{i-1,1}+\gamma_{i-1,2}+\gamma_{i-1,3}+\gamma_{i-1,4}\right)+\sum_{j=1}^{i-2} \frac{\partial \alpha_{j}}{\partial \widehat{\theta}} z_{j+1}^{3}\right)+\frac{\partial \alpha_{i-2}}{\partial \widehat{\theta}} \tau_{i-1}, \\
\tau_{i-1}\left(y, \widehat{x}_{1}, \ldots, \widehat{x}_{i-2}, \widehat{\theta}\right)= & \tau_{i-2}+\Gamma\left(\gamma_{i-1,1}+\gamma_{i-1,2}+\gamma_{i-1,3}+\gamma_{i-1,4}\right) z_{i-1}^{4},
\end{aligned}
$$

such that $V_{i-1}\left(e, \bar{z}_{i-1}, \widehat{\theta}\right)=(\delta / 2)\left(e^{T} P e\right)^{2}+(1 / 2 \Gamma) \widetilde{\theta}^{2}+\sum_{j=1}^{i-1}\left(z_{j}^{4} / 4\right)$ satisfies

$$
\begin{aligned}
\mathcal{L} V_{i-1} \leq & -\sum_{j=1}^{i-1} c_{j} z_{j}^{4}+\sum_{j=2}^{i-1} b_{j} z_{1}^{4}-v_{1}\left(z_{1}\right) z_{1}^{4}-a_{00}|e|^{4}+\sum_{j=1}^{i-1} a_{j 1} e_{1}^{4}+\Delta_{i-1}(|\eta|) \\
& +\bar{a}_{i-1,0} z_{i}^{4}+\left(\frac{1}{\Gamma} \tilde{\theta}-\sum_{j=1}^{i-2} \frac{\partial \alpha_{j}}{\partial \widehat{\theta}} z_{j+1}^{3}\right)\left(\dot{\hat{\theta}}-\tau_{i-1}\right)
\end{aligned}
$$

where $c_{i-1}>0$ are the designed parameters, $a_{i-1,0}, \bar{a}_{i-1,0}, a_{i-1,1}, b_{i-1}$ are some positive constants, and $\gamma_{i-1,1}, \gamma_{i-1,2}, \gamma_{i-1,3}, \gamma_{i-1,4}$ are smooth nonnegative functions. In the sequel, we will prove that Lemma 4.1 still holds for Step $i$. Choosing

$$
V_{i}\left(e, \bar{z}_{i}, \widehat{\theta}\right)=V_{i-1}\left(e, \bar{z}_{i-1}, \hat{\theta}\right)+\frac{1}{4} z_{i}^{4},
$$


with the use of (4.6), (4.7), (4.15), and (A.1), the ItÔ differential of $z_{i}$ is given as follows:

$$
\begin{aligned}
d z_{i}= & \left(\alpha_{i}+z_{i+1}+\Omega_{i}-\frac{\partial \alpha_{i-1}}{\partial y} \theta^{*} e_{1}-\frac{\partial \alpha_{i-1}}{\partial y} \varphi_{1}(\eta, x)-\frac{\partial \alpha_{i-1}}{\partial \hat{\theta}} \dot{\hat{\theta}}\right) d t \\
& -\frac{1}{2} \frac{\partial^{2} \alpha_{i-1}}{\partial y^{2}} \psi_{1}(\eta, x) \psi_{1}^{T}(\eta, x) d t-\frac{\partial \alpha_{i-1}}{\partial y} \psi_{1}(\eta, x) d w
\end{aligned}
$$

where $\Omega_{i}=k_{i} y-k_{i-1}\left(\widehat{x}_{1}+k_{1} y\right)-\sum_{j=1}^{i-2}\left(\partial \alpha_{i-1} / \partial \widehat{x}_{j}\right)\left(\widehat{x}_{j+1}+k_{j+1} y-k_{j}\left(\widehat{x}_{1}+k_{1} y\right)\right)-\left(\partial \alpha_{i-1} / \partial y\right)\left(\widehat{x}_{1}+\right.$ $k_{1} y$ ). Using (2.2) and (A.2)-(A.4), we arrive at

$$
\begin{aligned}
\mathcal{L} V_{i} \leq & -\sum_{j=1}^{i-1} c_{j} z_{j}^{4}+\sum_{j=2}^{i-1} b_{j} z_{1}^{4}-v_{1}\left(z_{1}\right) z_{1}^{4}-a_{00}|e|^{4}+\sum_{j=1}^{i-1} a_{j 1} e_{1}^{4}+\Delta_{i-1}(|\eta|)+\bar{a}_{i-1,0} z_{i}^{4} \\
& +\left(\frac{1}{\Gamma} \tilde{\theta}-\sum_{j=1}^{i-2} \frac{\partial \alpha_{j}}{\partial \widehat{\theta}} z_{j+1}^{3}\right)\left(\dot{\hat{\theta}}-\tau_{i-1}\right) \\
& +z_{i}^{3}\left(\alpha_{i}+\Omega_{i}-\frac{\partial \alpha_{i-1}}{\partial \hat{\theta}} \dot{\hat{\theta}}+z_{i+1}-\frac{\partial \alpha_{i-1}}{\partial y} \theta^{*} e_{1}-\frac{\partial \alpha_{i-1}}{\partial y} \varphi_{1}(\eta, x)-\frac{1}{2} \frac{\partial^{2} \alpha_{i-1}}{\partial y^{2}} \psi_{1}(\eta, x) \psi_{1}^{T}(\eta, x)\right) \\
& +\frac{3}{2} z_{i}^{2} \operatorname{Tr}\left\{\left(\frac{\partial \alpha_{i-1}}{\partial y}\right)^{2} \psi_{1}^{T}(\eta, x) \psi_{1}(\eta, x)\right\} .
\end{aligned}
$$

Now, we estimate the last five terms, respectively, in the right-hand side of (A.5). According to Assumption 3.1, (3.2), (4.7), and Lemma 2.6, there exist positive real numbers $a_{i 0}, \bar{a}_{i 0}, a_{i 1}$, $a_{i 2}, a_{i 3}, a_{i 4}, b_{i 2}, b_{i 3}, b_{i 4}$, smooth nonnegative functions $\gamma_{i 1}, \gamma_{i 2}, \gamma_{i 3}, \gamma_{i 4}$ such that

$$
\begin{gathered}
z_{i}^{3} z_{i+1} \leq a_{i 0} z_{i}^{4}+\bar{a}_{i 0} z_{i+1}^{4}, \quad-z_{i}^{3} \frac{\partial \alpha_{i-1}}{\partial y} \theta^{*} e_{1} \leq a_{i 1} e_{1}^{4}+\theta \gamma_{i 1}\left(\bar{z}_{i}\right) z_{i}^{4}, \\
-z_{i}^{3} \frac{\partial \alpha_{i-1}}{\partial y} \varphi_{1}(\eta, x) \leq\left|z_{i}\right|^{3}\left(1+\left(\frac{\partial \alpha_{i-1}}{\partial y}\right)^{2}\right)^{1 / 2}\left(\varphi_{10}(|\eta|)+\varphi_{11}\left(z_{1}\right)\right) \\
\leq a_{i 2} \varphi_{10}^{4}(|\eta|)+b_{i 2} z_{1}^{4}+\theta \gamma_{i 2}\left(\bar{z}_{i}\right) z_{i}^{4}, \\
-\frac{1}{2} \frac{\partial^{2} \alpha_{i-1}}{\partial y^{2}} z_{i}^{3} \psi_{1}(\eta, x) \psi_{1}^{T}(\eta, x) \leq\left|z_{i}\right|^{3}\left|\frac{\partial^{2} \alpha_{i-1}}{\partial y^{2}}\right|\left(\psi_{10}^{2}(|\eta|)+\psi_{11}^{2}\left(z_{1}\right)\right) \\
\leq a_{i 3} \psi_{10}^{4}(|\eta|)+b_{i 3} z_{1}^{4}+\theta \gamma_{i 3}\left(\bar{z}_{i}\right) z_{i}^{4}, \\
\frac{3}{2} z_{i}^{2} \operatorname{Tr}\left\{\left(\frac{\partial \alpha_{i-1}}{\partial y}\right)^{2} \psi_{1}^{T}(\eta, x) \psi_{1}(\eta, x)\right\} \\
\leq 3 z_{i}^{2}\left(\frac{\partial \alpha_{i-1}}{\partial y}\right)^{2}\left(\psi_{10}^{2}(|\eta|)+\psi_{11}^{2}\left(z_{1}\right)\right) \\
\leq a_{i 4} \psi_{10}^{4}(|\eta|)+b_{i 4} z_{1}^{4}+\theta \gamma_{i 4}\left(\bar{z}_{i}\right) z_{i}^{4} .
\end{gathered}
$$


Choosing $\alpha_{i}$ and $\tau_{i}$ as (4.17) and substituting (A.6) into (A.5), (4.18) holds, where $c_{i}>0$ is a design parameter,

$$
\Delta_{i}(|\eta|)=\Delta_{i-1}(|\eta|)+a_{i 2} \varphi_{10}^{4}(|\eta|)+a_{i 3} \psi_{10}^{4}(|\eta|)+a_{i 4} \psi_{10}^{4}(|\eta|), \quad b_{i}=b_{i 2}+b_{i 3}+b_{i 4} .
$$

\section{Acknowledgments}

This work was supported by National Natural Science Foundation of China (nos. 61104222 and 10971256), Natural Science Foundation of Jiangsu Province (no. BK2011205), and Natural Science Foundation of Jiangsu Normal University (no. 11XLR08).

\section{References}

[1] H. Deng, M. Krstić, and R. J. Williams, "Stabilization of stochastic nonlinear systems driven by noise of unknown covariance," IEEE Transactions on Automatic Control, vol. 46, no. 8, pp. 1237-1253, 2001.

[2] N. Duan and X.-J. Xie, "Further results on output-feedback stabilization for a class of stochastic nonlinear systems," IEEE Transactions on Automatic Control, vol. 56, no. 5, pp. 1208-1213, 2011.

[3] N. Duan, X.-J. Xie, and X. Yu, "State feedback stabilization of stochastic nonlinear systems with SiISS inverse dynamics," International Journal of Robust and Nonlinear Control, vol. 21, no. 16, pp. 1903-1919, 2011.

[4] N. Duan, X. Yu, and X.-J. Xie, “Output feedback control using small-gain conditions for stochastic nonlinear systems with SiISS inverse dynamics," International Journal of Control, vol. 84, no. 1, pp. 47-56, 2011.

[5] W.-Q. Li and X.-J. Xie, "Inverse optimal stabilization for stochastic nonlinear systems whose linearizations are not stabilizable," Automatica, vol. 45, no. 2, pp. 498-503, 2009.

[6] W. Li, X.-J. Xie, and S. Zhang, "Output-feedback stabilization of stochastic high-order nonlinear systems under weaker conditions," SIAM Journal on Control and Optimization, vol. 49, no. 3, pp. 1262 1282, 2011.

[7] L. Liu and X.-J. Xie, "Output-feedback stabilization for stochastic high-order nonlinear systems with time-varying delay," Automatica, vol. 47, no. 12, pp. 2772-2779, 2011.

[8] L. Liu and X. J. Xie, "State-feedback stabilization for stochastic high-order nonlinear systems with SISS inverse dynamics," Asian Journal of Control, vol. 14, no. 4, pp. 1-11, 2012.

[9] S.-J. Liu, J.-F. Zhang, and Z.-P. Jiang, "Decentralized adaptive output-feedback stabilization for largescale stochastic nonlinear systems," Automatica, vol. 43, no. 2, pp. 238-251, 2007.

[10] S.-J. Liu, J.-F. Zhang, and Z.-P. Jiang, "A notion of stochastic input-to-state stability and its application to stability of cascaded stochastic nonlinear systems," Acta Mathematicae Applicatae Sinica, vol. 24, no. 1, pp. 141-156, 2008.

[11] S.-J. Liu, J.-F. Zhang, and Z.-P. Jiang, “Global output-feedback stabilization for a class of stochastic non-minimum-phase nonlinear systems," Automatica, vol. 44, no. 8, pp. 1944-1957, 2008.

[12] Y. Liu and J. Zhang, "Minimal-order observer and output-feedback stabilization control design of stochastic nonlinear systems," Science in China F, vol. 47, no. 4, pp. 527-544, 2004.

[13] Y.-G. Liu and J.-F. Zhang, "Practical output-feedback risk-sensitive control for stochastic nonlinear systems with stable zero-dynamics," SIAM Journal on Control and Optimization, vol. 45, no. 3, pp. 885926, 2006.

[14] X. Mao, "A note on the LaSalle-type theorems for stochastic differential delay equations," Journal of Mathematical Analysis and Applications, vol. 268, no. 1, pp. 125-142, 2002.

[15] Z. Pan and T. Başar, "Backstepping controller design for nonlinear stochastic systems under a risksensitive cost criterion," SIAM Journal on Control and Optimization, vol. 37, no. 3, pp. 957-995, 1999.

[16] Z. Pan, Y. Liu, and S. Shi, "Output feedback stabilization for stochastic nonlinear systems in observer canonical form with stable zero-dynamics," Science in China F, vol. 44, no. 4, pp. 292-308, 2001. 
[17] Y. Shen, Q. Luo, and X. Mao, "The improved LaSalle-type theorems for stochastic functional differential equations," Journal of Mathematical Analysis and Applications, vol. 318, no. 1, pp. 134-154, 2006.

[18] J. Spiliotis and J. Tsinias, "Notions of exponential robust stochastic stability, ISS and their Lyapunov characterization," International Journal of Robust and Nonlinear Control, vol. 13, no. 2, pp. 173-187, 2003.

[19] C. Tang and T. Basar, "Stochastic stability of singularly perturbed nonlinear systems," in Proceedings of the 40th IEEE Conference on Decision and Control, pp. 399-404, Orlando, Fla, USA, 2001.

[20] J. Tian and X.-J. Xie, "Adaptive state-feedback stabilization for high-order stochastic non-linear systems with uncertain control coefficients," International Journal of Control, vol. 80, no. 9, pp. 1503 1516, 2007.

[21] J. Tsinias, "Stochastic input-to-state stability and applications to global feedback stabilization," International Journal of Control, vol. 71, no. 5, pp. 907-930, 1998.

[22] J. Tsinias, "The concept of "exponential input to state stability" for stochastic systems and applications to feedback stabilization," Systems E Control Letters, vol. 36, no. 3, pp. 221-229, 1999.

[23] Z. Wu, M. Cui, X. Xie, and P. Shi, "Theory of stochastic dissipative systems," IEEE Transactions on Automatic Control, vol. 56, no. 7, pp. 1650-1655, 2011.

[24] Z.-J. Wu, X.-J. Xie, and S.-Y. Zhang, "Stochastic adaptive backstepping controller design by introducing dynamic signal and changing supply function," International Journal of Control, vol. 79, no. 12, pp. 1635-1646, 2006.

[25] Z.-J. Wu, X.-J. Xie, and S.-Y. Zhang, “Adaptive backstepping controller design using stochastic smallgain theorem," Automatica, vol. 43, no. 4, pp. 608-620, 2007.

[26] Z.-J. Wu, X.-J. Xie, P. Shi, and Y.-Q. Xia, "Backstepping controller design for a class of stochastic nonlinear systems with Markovian switching," Automatica, vol. 45, no. 4, pp. 997-1004, 2009.

[27] X.-J. Xie, "A robust model reference adaptive control without strictly positive real condition," International Journal of Control, vol. 75, no. 14, pp. 1136-1144, 2002.

[28] X.-J. Xie and N. Duan, "Output tracking of high-order stochastic nonlinear systems with application to benchmark mechanical system," IEEE Transactions on Automatic Control, vol. 55, no. 5, pp. 11971202, 2010.

[29] X.-J. Xie, N. Duan, and X. Yu, "State-feedback control of high-order stochastic nonlinear systems with SiISS inverse dynamics," IEEE Transactions on Automatic Control, vol. 56, no. 8, pp. 1921-1926, 2011.

[30] X.-J. Xie and W.-Q. Li, "Output-feedback control of a class of high-order stochastic nonlinear systems," International Journal of Control, vol. 82, no. 9, pp. 1692-1705, 2009.

[31] X.-J. Xie and J. Tian, "State-feedback stabilization for high-order stochastic nonlinear systems with stochastic inverse dynamics," International Journal of Robust and Nonlinear Control, vol. 17, no. 14, pp. 1343-1362, 2007.

[32] X.-J. Xie and J. Tian, "Adaptive state-feedback stabilization of high-order stochastic systems with nonlinear parameterization," Automatica, vol. 45, no. 1, pp. 126-133, 2009.

[33] X.-J. Xie and S. Y. Zhang, "A robust adaptive pole-placement controller without strictly positive real condition," International Journal of Adaptive Control and Signal Processing, vol. 16, no. 1, pp. 39-59, 2002.

[34] X. Yu and X.-J. Xie, "Output feedback regulation of stochastic nonlinear systems with stochastic iISS inverse dynamics," IEEE Transactions on Automatic Control, vol. 55, no. 2, pp. 304-320, 2010.

[35] X. Yu, X.-J. Xie, and N. Duan, "Small-gain control method for stochastic nonlinear systems with stochastic iISS inverse dynamics," Automatica, vol. 46, no. 11, pp. 1790-1798, 2010.

[36] X. Yu, X.-J. Xie, and Y.-Q. Wu, "Further results on output-feedback regulation of stochastic nonlinear systems with SiISS inverse dynamics," International Journal of Control, vol. 83, no. 10, pp. 2140-2152, 2010.

[37] X. Yu, X.-J. Xie, and Y.-Q. Wu, "Decentralized adaptive output-feedback control for stochastic interconnected systems with stochastic unmodeled dynamic interactions," International Journal of Adaptive Control and Signal Processing, vol. 25, no. 8, pp. 740-757, 2011.

[38] E. D. Sontag, "Smooth stabilization implies coprime factorization," IEEE Transactions on Automatic Control, vol. 34, no. 4, pp. 435-443, 1989.

[39] E. D. Sontag, "Comments on integral variants of ISS," Systems \& Control Letters, vol. 34, no. 1-2, pp. 93-100, 1998.

[40] H. Ito and Z.-P. Jiang, “Necessary and sufficient small gain conditions for integral input-to-state stable systems: a Lyapunov perspective," IEEE Transactions on Automatic Control, vol. 54, no. 10, pp. 23892404, 2009.

[41] H. Ito, "A Lyapunov approach to cascade interconnection of integral input-to-state stable systems," IEEE Transactions on Automatic Control, vol. 55, no. 3, pp. 702-708, 2010. 


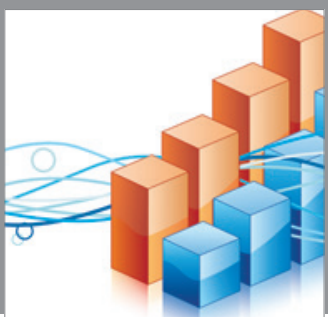

Advances in

Operations Research

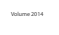

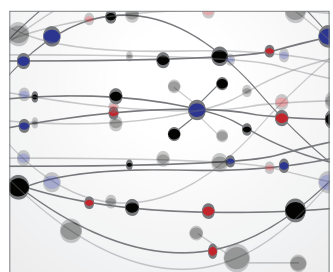

\section{The Scientific} World Journal
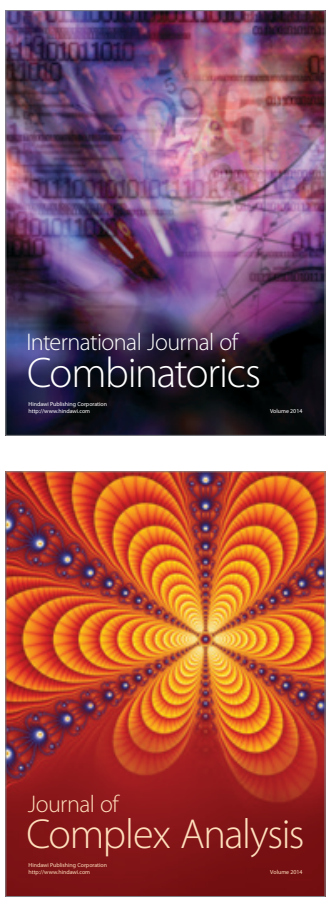

International Journal of

Mathematics and

Mathematical

Sciences
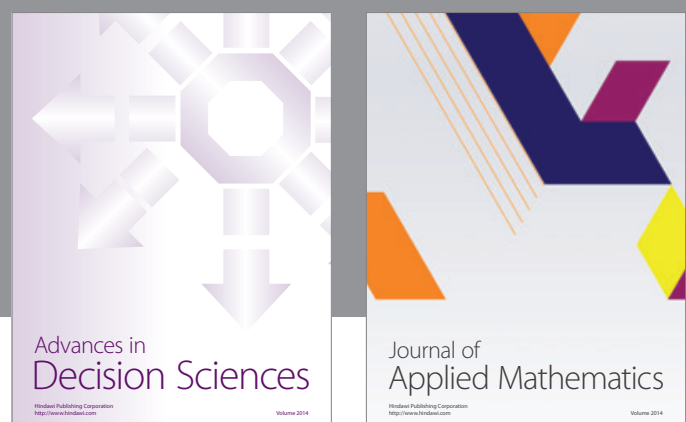

Journal of

Applied Mathematics
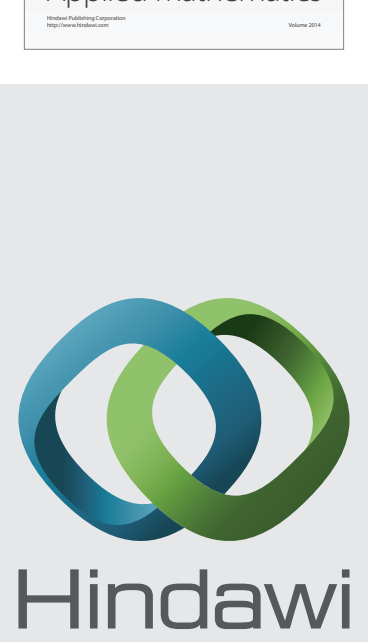

Submit your manuscripts at http://www.hindawi.com
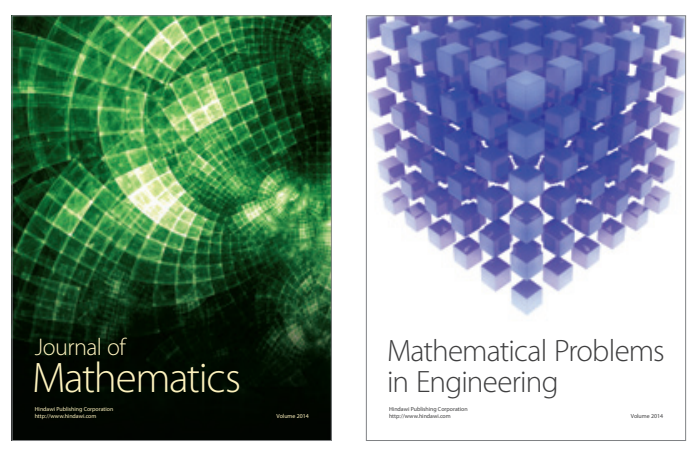

Mathematical Problems in Engineering
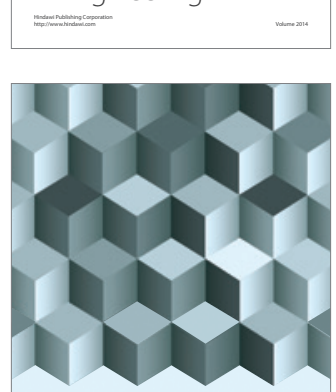

Journal of

Function Spaces
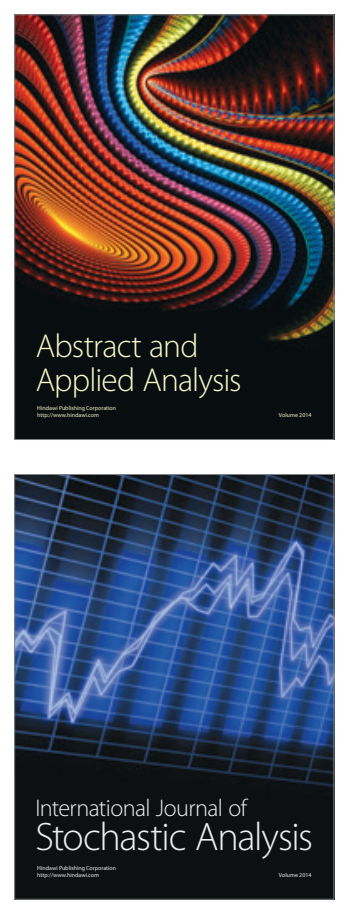

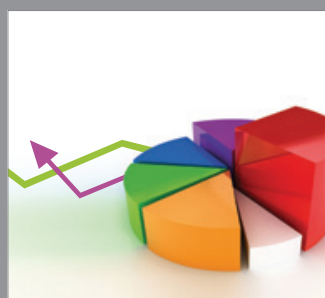

ournal of

Probability and Statistics

Promensencen
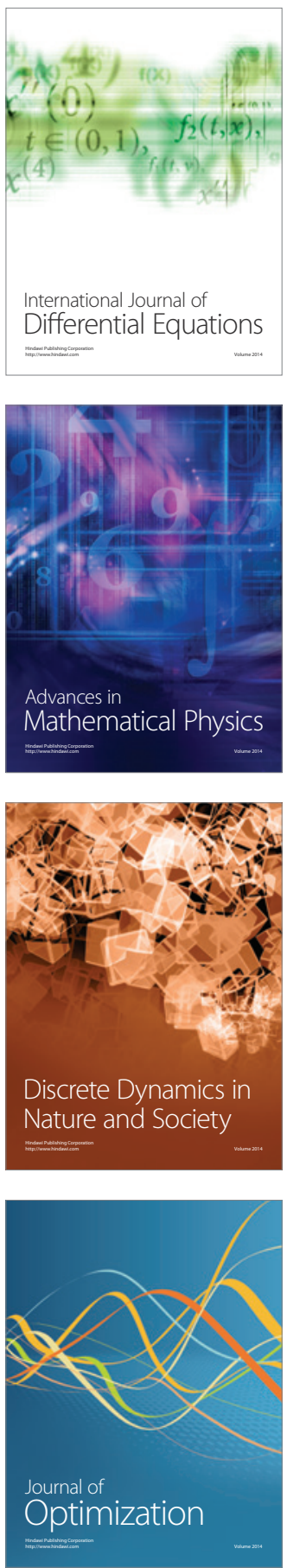